\title{
COMPARATIVE ANALYSIS OF IMPROVED HIGH PERFORMANCE DIRECT POWER CONTROL OF THREE PHASE PWM RECTIFIER
}

\author{
Hemlata S Pol ${ }^{1}$ \\ ${ }^{1}$ Department of Electronics Engineering, Fr Conceicao Rodrigues College of Engineering, Bandra,India
}

\begin{abstract}
Direct Power Control with switching table control produces irregular ripples and variable switching frequency. In the improved DPC of PWM rectifiers using fractional control period of the active voltage vector and the rest period for the null vector not only makes it line side inductance independent of the circuit and is very simple to implement due to the duty cycle concept. By simulation it is identified that the level of distortion as well as regulation of the DC link voltage with control of power factor improves the overall performance of the drive. The improved Direct Power Control (DPC) PWM methodology is comprehensively analyzed and studied for three phase rectifiers and compared with the classical DPC with PLL. The MATLAB simulations shows the effectiveness in obtaining unity power factor and constant dc link voltage control. The power ripples are considerably reduced and input sinusoidal grid currents are obtained.
\end{abstract}

Keywords - Direct power control, instantaneous active and reactive power, pulse width modulation, duty cycle, dc link control, unity power factor

\section{INTRODUCTION}

The application of three phase rectifiers in areas like renewable energy systems as wind turbines and photovoltaic, FACTS, drives ac and dc, battery storage and micro grid operations have increased tremendously. There are various models for power control of PWM rectifier like voltage oriented control (VOC), voltage based DPC, virtual flux oriented control (VF-VOC), VF-DPC. Voltage oriented based control decomposes grid currents into active and reactive power components separately which through PI controllers are fed through modulator to synthesize voltage vectors. Fine tuning of PI is required to achieve steady and dynamic response.

In DPC for achieving satisfactory performance the switching frequency required is very high, which adds up to the hardware burden. Major work has been done to tackle this problem such as SVM-based DPC, predictive control, fuzzy logic control and deadbeat control etc. But these methods further increased the complexity and computational burden. To address the problem several techniques with new switching tables were proposed. But they tried to incorporate accuracy and efficiency by eliminating ac voltage sensors, which reduced the overall system robustness.

Conclusively the improved DPC that is studied here does not focus on effectiveness of switching tables rather it focuses on improving steady performance. The parameter being selected for this is duty cycle of the voltage vectors. The concept of duty cycle control is to select the fraction of time for which the voltage vector will be applied. The existing duty cycle methods were parameter dependent, which was again contributing to decreasing robustness and increasing complexity. The improved method is kept simple by eliminating the requirement of system parameters. Simulation results prove that the new improved DPC has high performance .

Direct power control is high performance instantaneous power control theory basically similar to direct torque control in motor drives. It directly selects the desired grid voltage vector from predefined switching table according to the grid voltage position or virtual flux position and the errors between the reference and feedback powers are calculated. Conventional DPC has a drawback of high power ripples and variable switching frequency. The improved DPC introduces the concept of fractional control of duty cycle by active vectors over the allocated period improves the performance of the rectifier by making it independent of line inductances thus robustness is achieved and ripples in power are also reduced with almost unity power factor operation.

Unique features offered by this improved DPC are:

- $\quad$ Fixed and low switching frequency.

- Sampling frequency for digital implementation is low.

- Parameter independent thus robust.

- Simple and easy control with only two voltage vectors.

\section{PRINCIPLE OF DPC IN THREE PHASE PWM RECTIFIER}

The topology as seen from the fig. 1 which is two level can be mathematically modelled into two phase stationary reference $(\alpha \beta)$ frame and with $\mathrm{R}$ and $\mathrm{L}$ as equivalent series resistance and choke. 
The Direct Power Control (DPC) is based on the instantaneous active and reactive power control loop. There are no internal current control loop and no PWM modulator block. The switching states are determined with a switching table based on the instantaneous errors between the commanded and estimated values of active and reactive power.

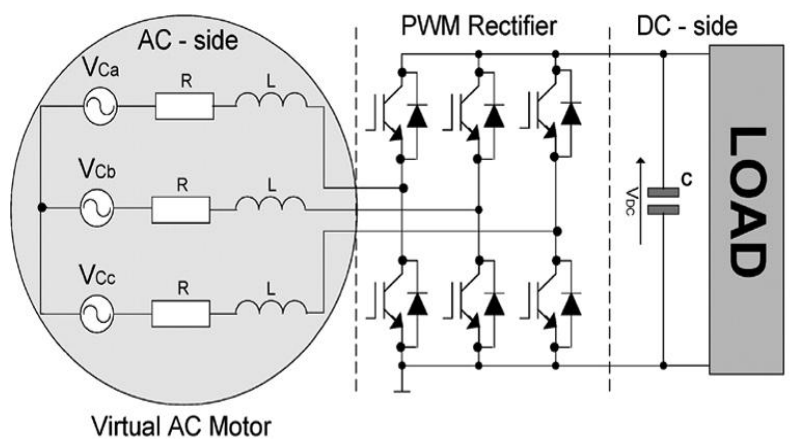

Fig.1 Three phase rectifier topology [1]

Fig. 2 shows the conventional classical configuration of DPC where the instantaneous active and reactive powers are the controlled variables, this method is based on switching table for selecting the desired voltage vector. The drawback of $\mathrm{DPC}$ is the variable switching frequency, system parameters and the switching table.

The PI controller needs fine tuning and the power ripples along with harmonic distortion in the input side cannot be filtered out easily with this basic configuration. In the new improved DPC with duty cycle adjustment in fractional control environment not only improves the overall performance but it reduces the complexity of various system parameters.

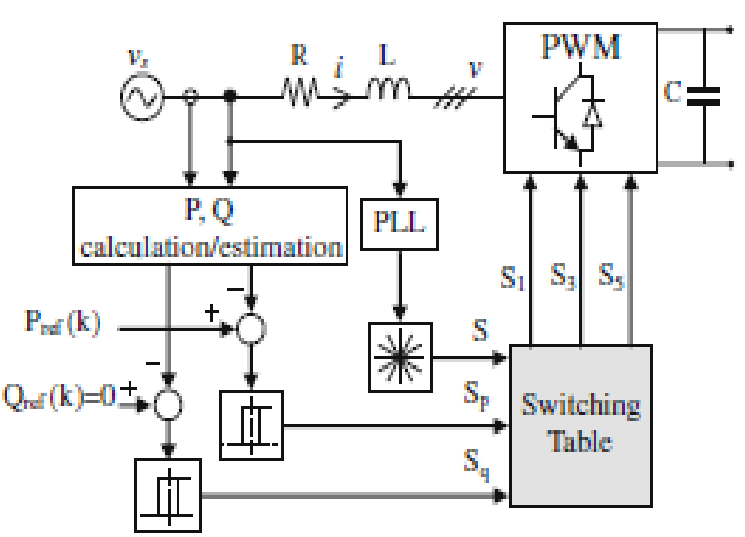

Fig.2 Classical configuration of DPC with PLL

According to the switching table first time as usedby Noguchi et al for the control scheme as depicted in fig.2. This method is based on selecting a voltage vector from lookup table, table 1 , according to the errors of active and reactive powers as well as the angular position of the source voltage vector.
The drawback of the DPC is variable switching frequency which depends on the sampling frequency, the switching table , system parameters, reference values of active and reactive powers and hysteresis band controllers and the switching states. This variable frequency introduces harmonic spectrum in $\mathrm{AC}$ line currents and thus the design of filters become very difficult. In order to attenuate the harmonics and power ripples large value of sampling frequency and inductance should be selected this increases the cost, losses and reduces system dynamics.

There have been many approaches to control these factors as virtual flux estimaters but PI controller tunning is very complex and thus it becomes difficult to achieve high dynamic performance.

\section{IMPROVED DIRECT POWER CONTROL WITH FRACTIONAL CONTROL}

The improved direct power control method has advantage of:

- Simple algorithm for duty cycle determination

- Independent of line inductance parameter

- Operation at constant frequency simple filter design

- Low switching and sampling frequency

- Calculations are fast and simplified

The equation for grid voltage ' $e$ ' can be written as :

$$
e=\mathrm{R} i+\mathrm{L} \frac{d i}{d t}+\mathrm{v}
$$

Where $v, e, i$ represent rectifier voltage vector, grid voltage vector and grid current vector respectively.

Transforming the three-phase model to stationary $(\alpha \beta)$ frame, the complex power $\mathrm{S}$ for active power $\mathrm{P}$ and reactive power $\mathrm{Q}$ can be given as :

Complex power as per [1]

$$
\begin{aligned}
& \mathrm{S}=\mathrm{P}+\mathrm{jQ}=1.5\left(\mathrm{i}^{*} \mathrm{e}\right) \\
& \text { Instantaneous active power } \mathrm{p}=\mathrm{u} \alpha \mathrm{i} \alpha+\mathrm{u} \beta \mathrm{i} \beta \\
& \text { Instantaneous reactive power } \mathrm{q}=\mathrm{u} \beta \mathrm{i} \alpha-\mathrm{u} \alpha \mathrm{i} \beta
\end{aligned}
$$

The equation for grid voltage ' $\mathrm{e}$ ' is :

$$
\mathrm{e}=|\mathrm{e}|^{e^{j w t}}
$$




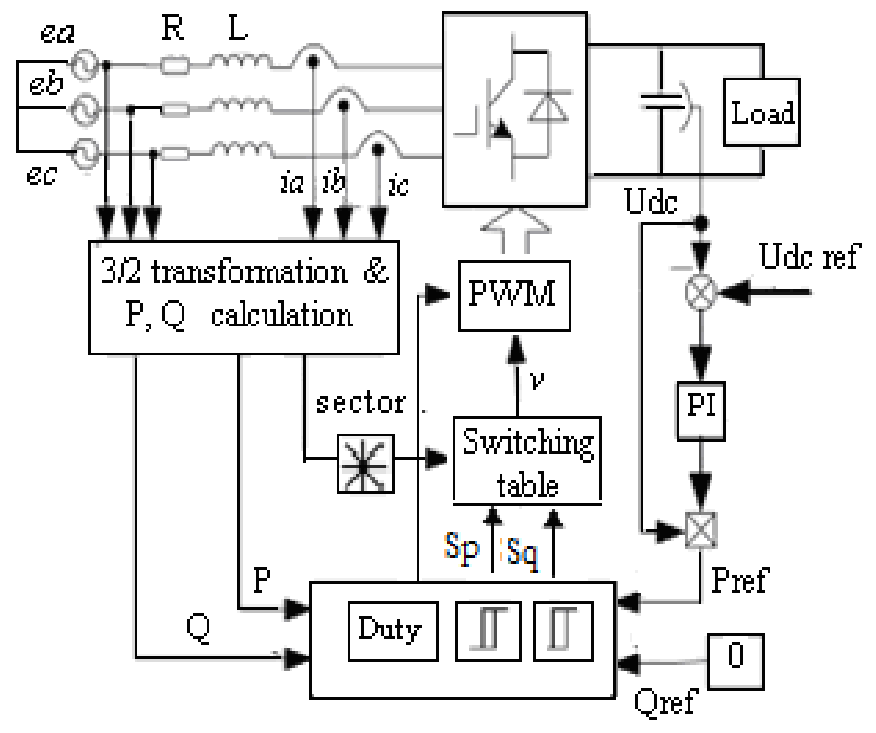

Fig.3 Improved Duty Cycle based DPC [1]

Differentiating e we get :

$$
\frac{d e}{d t}=j w|e|^{e^{j w t}}=j w e
$$

Differentiating the grid current $i$ :

$$
\frac{d i}{d t}=\frac{1}{L}(e-v-\mathrm{R} i)
$$

Now differentiating complex power :

$$
\begin{aligned}
& \frac{d S}{d t}=\frac{1}{L}\left[1.5\left(|\mathrm{e}|^{2}-\mathrm{v}^{*} \mathrm{e}\right)-(\mathrm{R}-\mathrm{j} \mathrm{w} \mathrm{L}) . \mathrm{S}\right] \\
& \frac{d P}{d t}=\frac{3}{2 * L}\left[|\mathrm{e}|-\operatorname{Re}\left(\mathrm{v}^{*} \mathrm{e}\right)\right]-\left(\frac{R}{L}\right) P-w q \\
& \frac{d Q}{d t}=-\frac{3}{2 * L}\left(\operatorname{Im}\left(v^{*} e\right)-\left(\frac{R}{L}\right) Q+w p\right)
\end{aligned}
$$

The expressions can be further simplified as :

$$
\begin{gathered}
\frac{d P}{d t}=\frac{3}{2 * L}|\mathrm{e}| 2-\frac{V d c}{L}|\mathrm{e}| \\
\cos \left(w t-\frac{\pi(\mathbf{n}-\mathbf{1})}{3}\right) \frac{R}{L} P
\end{gathered}
$$

$$
\frac{d Q}{d t}=-\frac{3}{2 * L} \frac{V d c}{L}|\mathrm{e}| \sin \left(w t-\frac{\pi(\mathbf{n}-\mathbf{1})}{3}\right)+\mathrm{w} p
$$

here Vdc is DC-link voltage. The power slopes can be calculated from eq. 9 and 10 .The rectifier voltage vectors with 6 sector division are as shown below.
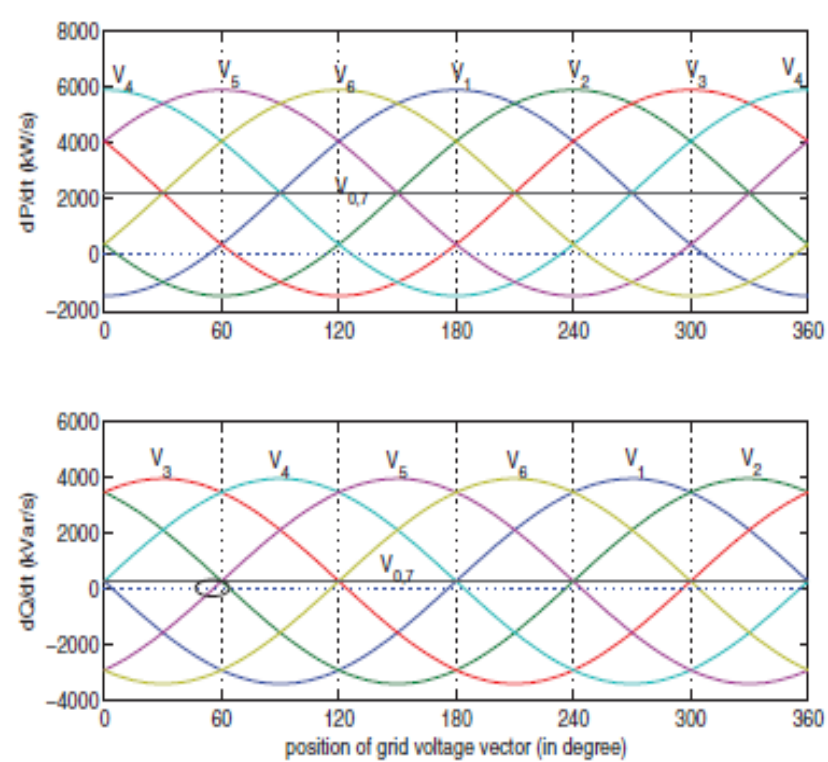

Fig.4 slopes of active power and reactive power verses grid vector position for various rectifier voltage vectors (assuming

$$
\mathrm{p}=900 \mathrm{~W} \text { and } \mathrm{q}=0 \text { ) [1] }
$$

According to the influences of voltage vectors over active and reactive power slopes in any sector $\mathrm{k}$ can be obtained as shown in table 1 where $\mathrm{k}$ is the cycling index .For controlling the active and reactive power simultaneously we can select specific set of vectors corresponding to incremental or decremental nature of $\mathrm{P}$ or $\mathrm{Q}$.

Based on the analysis of appropriate set of combinations of voltage vectors, it is imperative to notice that switching tables based on grid voltage vectors have some inherent drawbacks especially in wide power range.

The efforts to improve the selection of appropriate vector is done as per the following model. The six sectors on stationary reference frame and the rectifier voltage vectors are presented in fig. 5

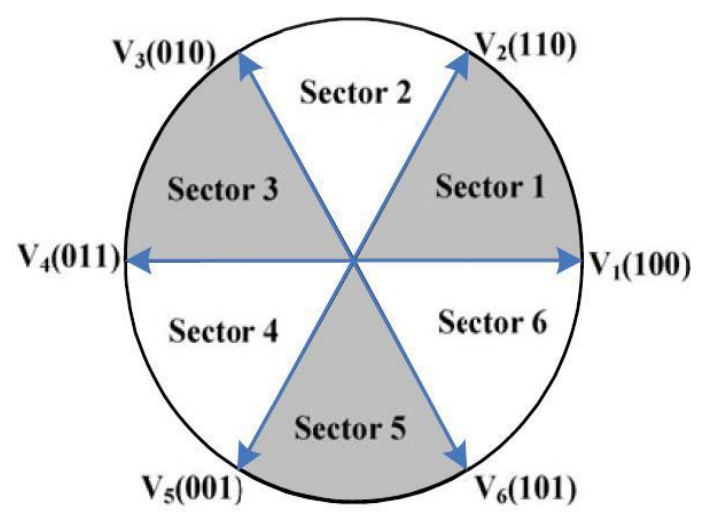

Fig. 5 Rectifier voltage vectors and sector division of DPC.[1] 
For simultaneous control of both active and reactive power the table. 1 can be summarized. It can be seen that there are more than two vectors to satisfy a change in power requirement. For example if both $\mathrm{P}$ and $\mathrm{Q}$ are increasing then V0,7 produces least variation on $\mathrm{Q}$, small (first half sector) or medium (second half sector) variation on $\mathrm{P} . \mathrm{Vk}+3$ has the biggest influence on $\mathrm{P}$ and medium on $\mathrm{Q}$. The influence of $\mathrm{Vk}+2$ is moderate.

Similarly for $\mathrm{P}$ increasing and $\mathrm{Q}$ decreasing there are insignificant differences between $\mathrm{Vk}-1$ and $\mathrm{Vk}$-2except that when transition from sector $\mathrm{k}$ to $\mathrm{k}+1, \mathrm{Vk}-2$ increases $\mathrm{Q}$ rather than decreasing with a value smaller than that caused by null vector.

Table 1: Switching table for DPC of PWM rectifier

\begin{tabular}{|l|l|l|}
\hline $\mathrm{Sp}$ & $\mathrm{Sq}$ & Selected vector \\
\hline 1 & 1 & $\mathrm{~V} 0,7, \mathrm{Vk}+2, \mathrm{Vk}+3$ \\
\hline 1 & 0 & $\mathrm{Vk}-1, \mathrm{Vk}-2$ \\
\hline 0 & 1 & $\mathrm{Vk}+1$ \\
\hline 0 & 0 & $\mathrm{Vk}$ \\
\hline
\end{tabular}

Table 2: Comparison of vectors in case of $\mathrm{P}$ and $\mathrm{Q}$ increasing[1]

\begin{tabular}{|l|l|l|}
\hline & $|\Delta \mathrm{P}|$ & $|\Delta \mathrm{Q}|$ \\
\hline $\mathrm{V} 0,7$ & Small to medium & small \\
\hline $\mathrm{Vk}+2$ & Medium to small & big \\
\hline $\mathrm{Vk}+3$ & big & medium \\
\hline
\end{tabular}

Table 3: Comparison of vectors in case of $\mathrm{P}$ increasing and $\mathrm{Q}$ decreasing [1]

\begin{tabular}{|l|l|l|}
\hline & $|\Delta \mathrm{P}|$ & $|\Delta \mathrm{Q}|$ \\
\hline Vk-1 & big & small \\
\hline Vk-2 & small & big \\
\hline
\end{tabular}

One special case of table 1 is selected to obtain active vector as shown in table 4. Similar results can be obtained from the other vectors also. Table 4 is used to study the comparison of DPC models.

Table 4: Active vector selection for the improved DPC with duty cycle control

\begin{tabular}{|l|l|l|}
\hline $\mathrm{Sp}$ & $\mathrm{Sq}$ & Selected vector \\
\hline 1 & 1 & $\mathrm{Vk}+3$ \\
\hline 1 & 0 & $\mathrm{Vk}-1$, \\
\hline 0 & 1 & $\mathrm{Vk}+1$ \\
\hline 0 & 0 & $\mathrm{Vk}$ \\
\hline
\end{tabular}

Duty cycle is the ratio of the applied duration of the active vector to the whole period and for duty cycle $=1$ the improved DPC will act as STDPC. Since active power is depending on the fractional control of duty cycle ' $d$ ' it will try to improve steady performance of the rectifier .From equation (6) slopes of the active power for the active vector s1 and for null vector s2 can be obtained. A typical waveform employing active as well as null vector is as shown

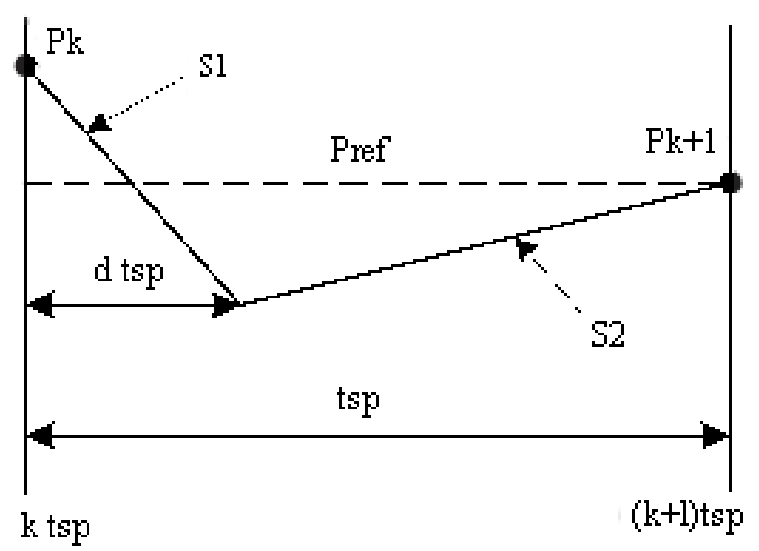

Fig 6 waveform of active power when both active and null vector is employed for one period [1]

Supposing that $\mathrm{P}$ reaches its reference value in a deadbeat fashion, the equation for $\mathrm{P}^{\mathrm{k}+1}$ can be :

$$
P^{k+1}=P^{k}+\text { s1.d.tsp }+ \text { s2.(1-d).tsp }
$$

i.e.

$$
P^{\text {ref }}=P^{k}+\text { s1.d.tsp }+ \text { s2.(1-d).tsp }
$$

from (11) \& (12) the optimized duty cycle d can given as :

$$
d=\frac{p^{r e f}-P^{k}-s 2 . t s p}{(s 1-s 2) t s p}
$$

The above equation for duty cycle requires accurate knowledge of slopes s1 and s2 of active powers. Unlike equation (6) where the power slopes were dependant on information of input inductance and resistance, this techniques doesn't require them . Hence trying to maintain the system simplicity and robustness . By expanding eq (13) following equation can be obtained :

$$
\mathrm{d}=\frac{\operatorname{Pr} e f-P k}{(s 1-s 2) t s p}+\frac{-s 2 t s p}{(s 1-s 2) t p}
$$

Considering the denominator to be constant the parameter dependence is eliminated in first term, whereas second term is complex and parameter dependent term. Here the numerator $\mathrm{s} 2$ is active power slope caused by the null vector which has small but constant influence over the reactive power. Hence the first term can reflects the regulation of active power whereas the second reflects regulation of reactive power .

Thus the final expression for this algorithm can be given as :

$$
d=\left|\frac{\operatorname{Pr} e f-P k}{C p}\right|+\left|\frac{Q r e f-Q k}{C q}\right|
$$


Based on the variations in $\mathrm{Cp}$ and $\mathrm{C}_{\mathrm{q}}$ the accuracy of the duty cycle $\mathrm{d}$ influence the steady performance and dynamic response only partly, but it would not cause much influence on the stability of system, because the mechanism of STDPC still works to ensure the system is stable. DPC has high performance control logic for PWM rectifier based on instantaneous power theory which was first proposed by Akaji in his paper.

Extensive simulations showed that to achieve good steady state and dynamic performance

$$
\mathrm{Cp}=\mathrm{Cq}=\frac{V d c}{L} \mathrm{e}^{\text {peak }} \mathrm{tsp}
$$

Where $\mathrm{e}^{\text {peak }}$ is the peak phase value of grid voltage. The basic principle of DPC is similar to direct torque control (DTC) in motor drives it directly selects the desired voltage vector from a switching table which is already defined, according to the grid voltage position (or virtual flux position) and the errors between the feedback value and reference active/reactive power value. In voltage oriented control the internal current loop is eliminated in DPC. As a result, DPC features very quick dynamic response with simple structure.

\section{SIMULATION RESULTS}

A digital computer simulation model is developed in MATLAB/SIMULINK platform to verify the effectiveness of the control method under steady state conditions. The waveforms obtained confirm the improvement in the DPC with minimum distortion and less harmonic noises ie. THD . The simulations are tested for two different sampling frequency of $20 \mathrm{KHz}$ and $40 \mathrm{KHz}$ and the effect of variation in the result can be observed significantly.

The system parameters used for simulations are Line resistance $\mathrm{R}=0.3 \Omega, \mathrm{L}=10 \mathrm{mH}, \mathrm{Vdc}=300 \mathrm{~V}, \mathrm{DC}$ bus capacitor $=2350 \mu \mathrm{F}$, active power constant gain $\mathrm{Cp}=183.7$ $\mathrm{W}$, Reactive power constant gain $\mathrm{Cq}=183.7 \mathrm{Var}$, $\mathrm{fs}=50$ $\mathrm{Hz}, \mathrm{tsp}=50 \mu \mathrm{sec}$

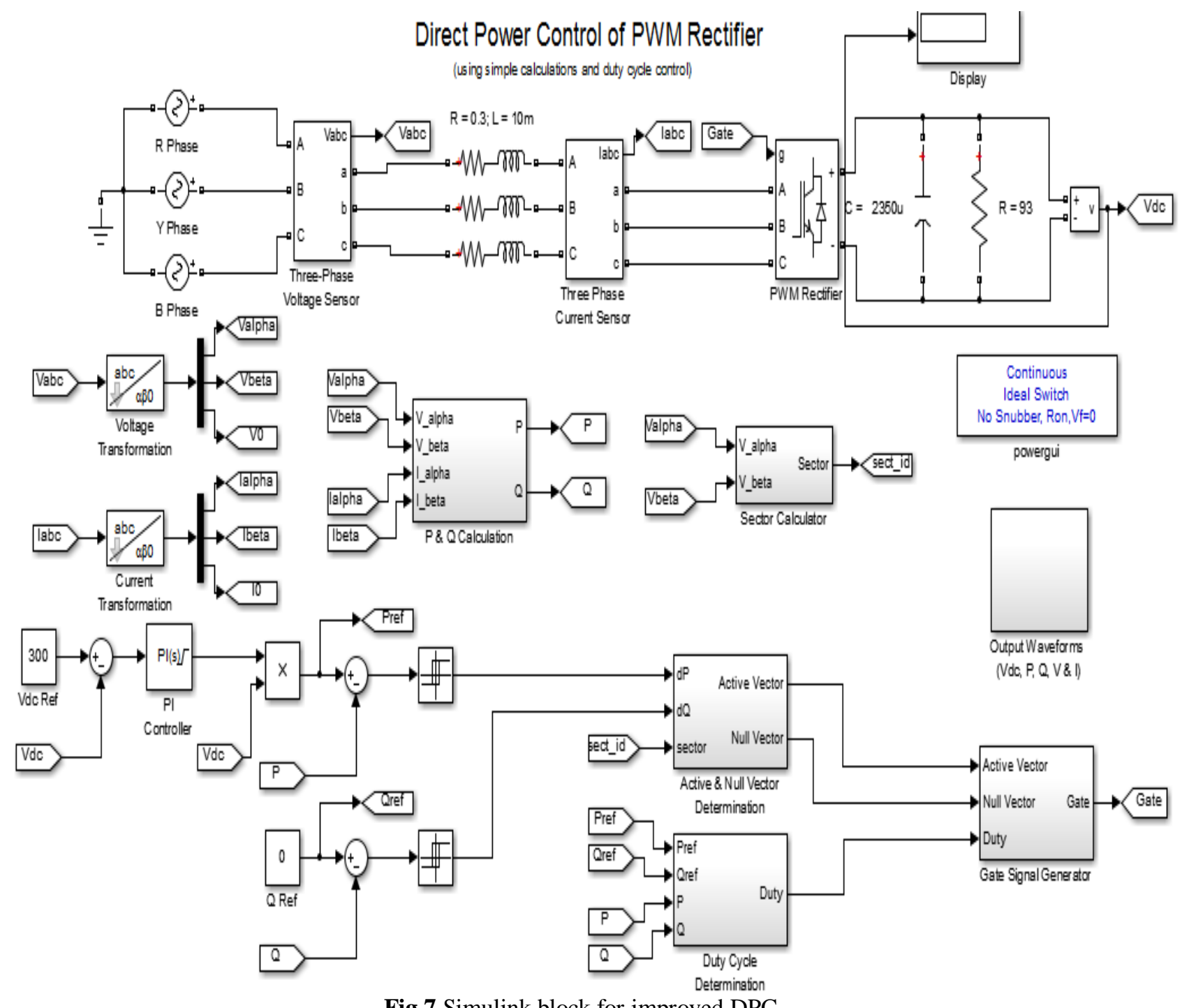

Fig 7 Simulink block for improved DPC 


\section{Link Voltage}

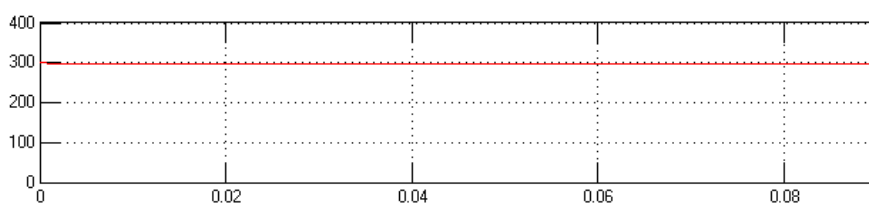

Active Power

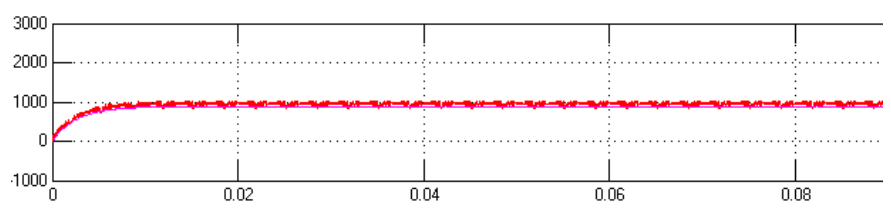

\section{Reactive Power}

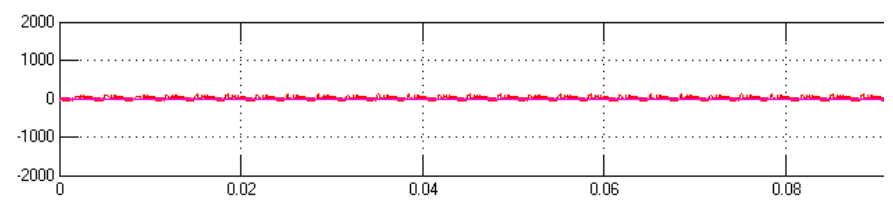

Voltage \& Current of Phase A
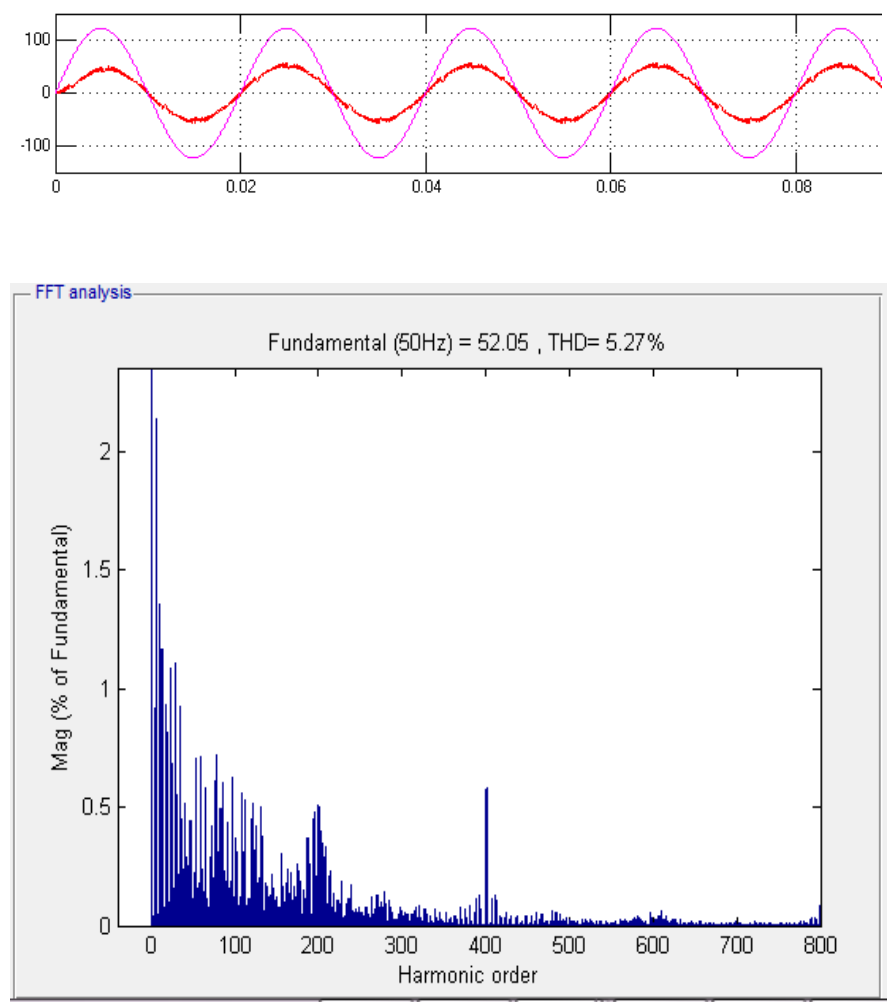

Fig 8 simulation of DPC with $20 \mathrm{Khz}$ sampling frequency

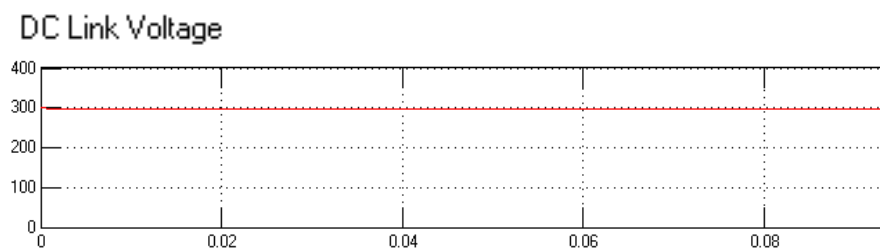

\section{Active Power}

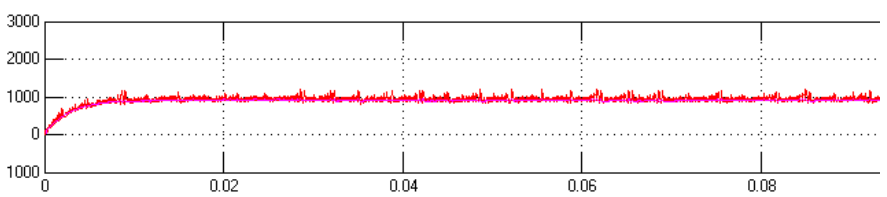

\section{Reactive Power}

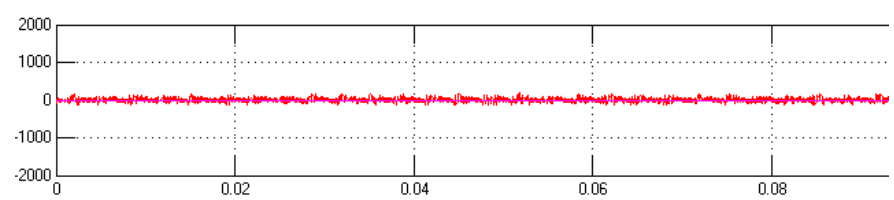

Voltage \& Current of Phase A
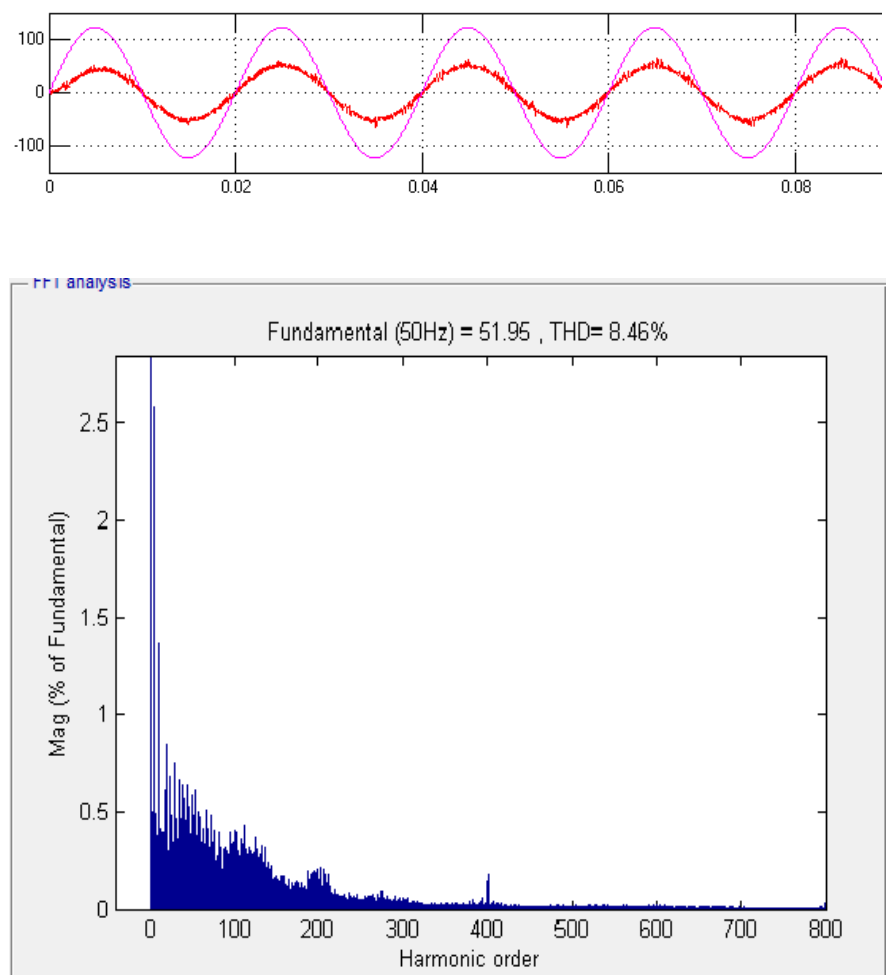

Fig 9 simulation of DPC with $40 \mathrm{Khz}$ sampling frequency 


\section{Link Voltage}

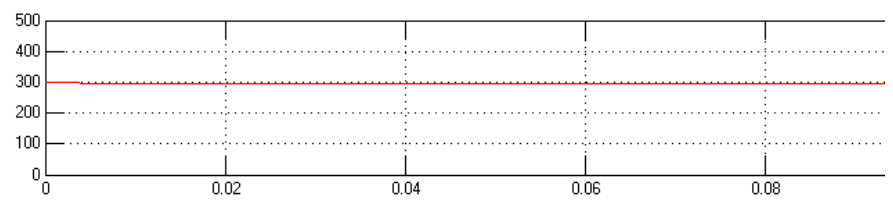

\section{Active Power}

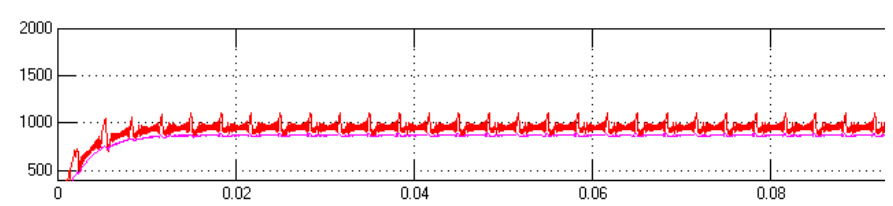

\section{Reactive Power}

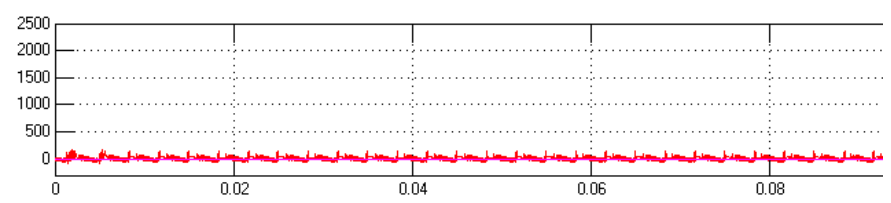

Voltage \& Current of Phase A
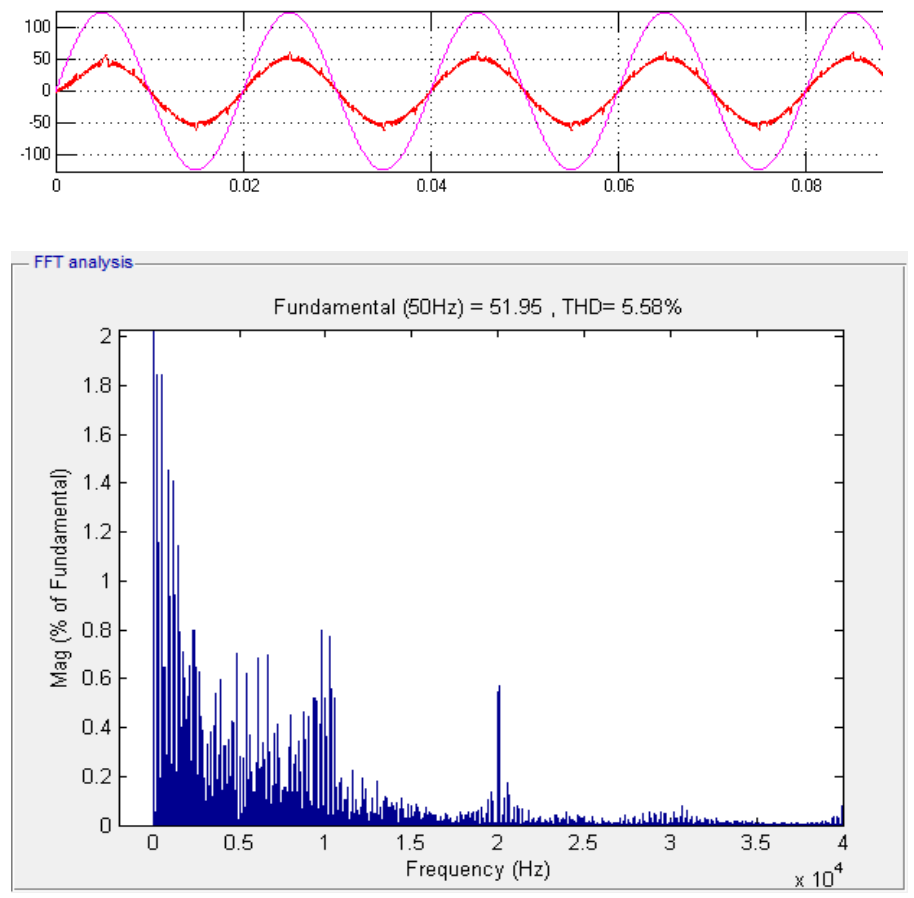

Fig. 10 simulation of DPC with PLL at $20 \mathrm{KHz}$

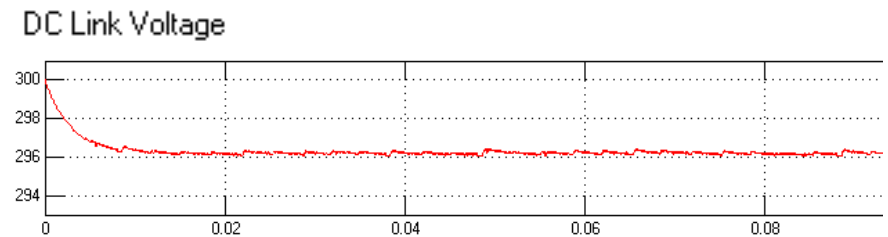

\section{Active Power}

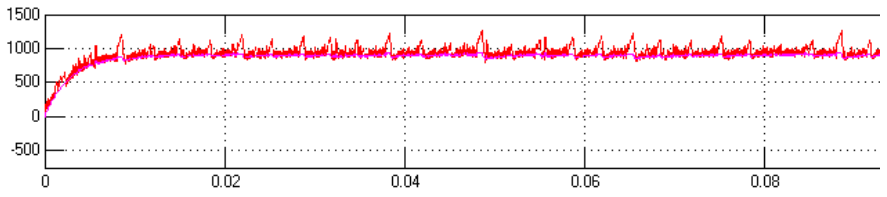

\section{Reactive Power}

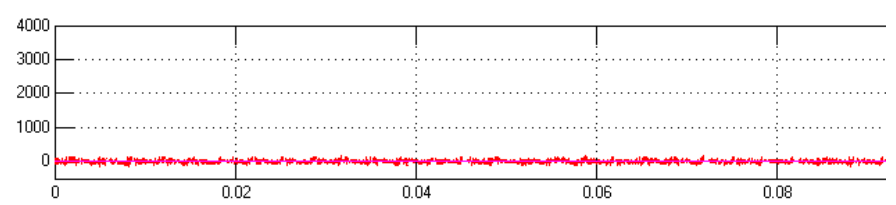

Voltage \& Current of Phase A
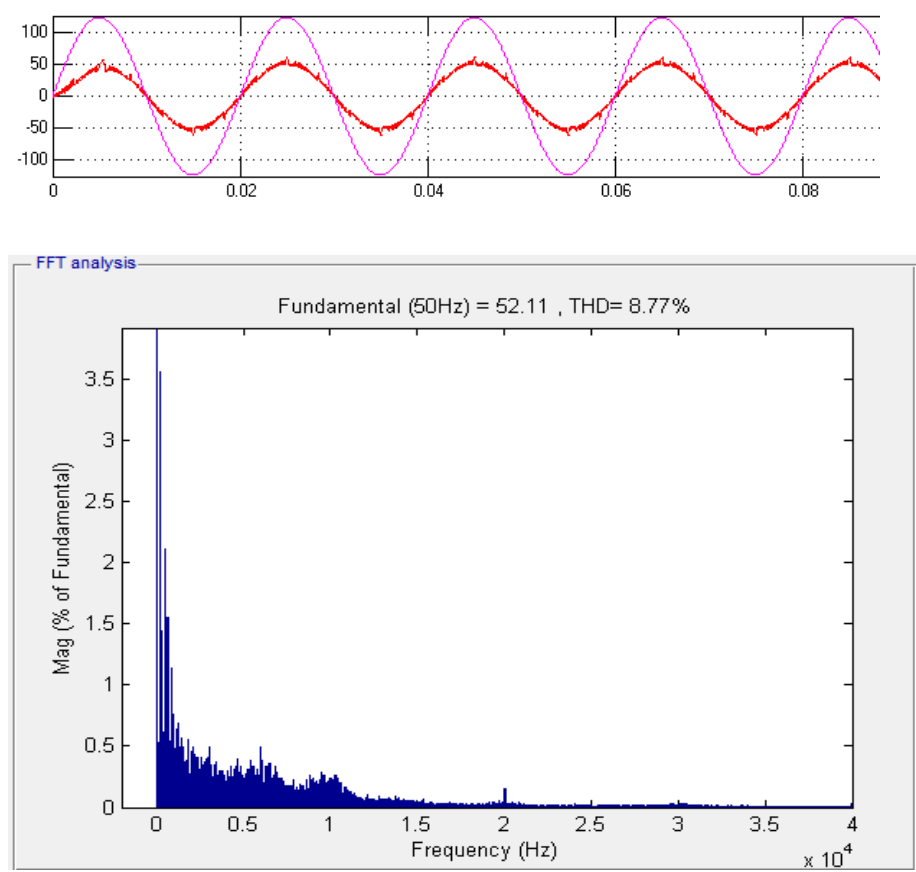

Fig. 11 simulation of DPC with PLL at $40 \mathrm{KHz}$ 
Table 5: THD $i$ for different values of sampling frequency

\begin{tabular}{|l|l|l|}
\multicolumn{4}{|c}{ (Q ref $=0$, P ref $=900 \mathrm{~W})$} \\
\hline \begin{tabular}{ll|l|} 
Configuration \\
Sampling frequency
\end{tabular} & $\begin{array}{l}\text { Classical } \\
\text { DPC with } \\
\text { PLL } \\
\text { THD } \%\end{array}$ & $\begin{array}{l}\text { Improved } \\
\text { THD } \%\end{array}$ \\
\hline DPC1 $20 \mathrm{KHz}$ & 5.58 & 5.27 \\
\hline DPC2 $40 \mathrm{KHz}$ & 8.77 & 8.46 \\
\hline
\end{tabular}

\section{CONCLUSION}

A DPC with simple approach of with duty cycle control was simulated and studied for harmonic distortion, power factor, switching losses and sinusoidal input ripple. It is found that using Direct Power Control and its controlled algorithm the performance of the rectifier greatly improves due to less harmonic distortion, good power factor, less switching loss and sinusoidal input current waveform. The main advantage of using duty cycle in DPC is that the rectifier parameters are independent of the control and only a fraction of controlperiod is used. Also the methodology used in DPC is of instantaneous active and reactive power theory which are regulated separately so the control does not require inner current loop as required for voltage oriented control thus giving high dynamic performance.

\section{REFERENCES}

[1]. "Performance Improvement of Direct Power Control of PWM Rectifier With Simple Calculation," Yongchang Zhang, Member, IEEE, Zhengxi Li, Senior Member, IEEE, Yingchao Zhang, Wei Xie, Zhengguo Piao, and Changbin $\mathrm{Hu}$ , IEEE TRANSACTIONS ON POWER ELECTRONICS, VOL. 28, NO. 7, JULY 2013

[2]. "Model Predictive Direct Power Control of a PWM Rectifier With Duty Cycle Optimization" Yongchang Zhang, Member, IEEE, Wei Xie, Zhengxi Li, Senior Member, IEEE, and Yingchao Zhang, IEEE TRANSACTIONS ON POWER ELECTRONICS, VOL. 28, NO. 11, NOVEMBER 2013

[3]. “ Predictive Direct Power Control of Three-Phase Pulsewidth Modulation (PWM) Rectifier Using Space-Vector Modulation (SVM)" Abdelouahab Bouafia, Jean-Paul Gaubert, Member, IEEE, and Fateh Krim, Senior Member, IEEE , IEEE TRANSACTIONS ON POWER ELECTRONICS, VOL. 25, NO. 1, JANUARY 2010

[4]". DPC of a Three Phase Rectifier Based on Positive Sequence

Detection"PanfiloR.MartinezRodriguez,Member,IEEE, Gerar doEscobar,SeniorMember,IEEE,AndresA.ValdezFernandez, Member,IEEE,MichaelHernandezGomez, andJoseM.Sosa, Me mber,IEEE I EEE TRANSACTIONS ON INDUSTRIAL ELECTRONICS,VOL.61,NO.8,AUGUST 2014

[5]. "Optimum Space Vector Computation Technique for Direct Power Control" Jose A. Restrepo, Member, IEEE, Jose M. Aller, Julio C. Viola, Alexander Bueno, and Thomas G. Habetler, Fellow, IEEE IEEE TRANSACTIONS ON POWER ELECTRONICS, VOL. 24, NO. 6, JUNE 2009
[6]. "Comparative study of SVPWM (space vector pulse width modulation) \& SPWM (sinusoidal pulse width modulation) based three phase voltage source inverters for variable speed drive" ICSI CCST 2013 IOP Publishing IOP Conf. Waheed Ahmedl,2 and Syed M Usman Alil 1 Department of Electronic Engineering, NED University of Engineering \& Technology, University Road, Karachi 75270, Pakistan. Series: Materials Science and Engineering $51(2013)$

[7]. Simple Direct Power Control of Three-Phase PWM Rectifier Using Space-Vector Modulation (DPC-SVM) Mariusz Malinowski, Member, IEEE, Marek Jasinski, Student Member, IEEE, and Marian P. Kazmierkowski, Fellow, IEEE IEEE TRANSACTIONS ON INDUSTRIAL ELECTRONICS, VOL. 51, NO. 2, APRIL 2004

[8]. Harmonic Mitigation Using 12-Pulse AC-DC Converter in Vector-Controlled Induction Motor Drives Bhim Singh, Senior Member, IEEE, G. Bhuvaneswari, Senior Member, IEEE, and Vipin Garg, Member, IEEE 